\title{
Influence du couvert de Eucalyptus camaldulensis (dehn) sur la diversité spécifique des herbacées dans la zone de Diembéring (Basse Casamance, Sénégal)
}

\author{
Arfang Ousmane Kémo GOUDIABY ${ }^{1,2^{*}}$, Siré Diédhiou SALL ${ }^{1}$, Ismaïla COLY ${ }^{1}$, \\ Saliou DJIBA ${ }^{2}$, Ngor NDOUR ${ }^{1}$ et Ibrahima NDOYE ${ }^{3}$ \\ ${ }^{1}$ Université Assane SECK de Ziguinchor, Faculté des Sciences et Technologies, \\ Laboratoire d'Agroforesterie et d'Ecologie, Département d'Agroforesterie, Sénégal. \\ ${ }^{2}$ Institut Sénégalais de Recherches Agricoles (ISRA/CRA de Djibélor), Sénégal. \\ ${ }^{3}$ Université Cheikh Anta Diop de Dakar, Faculté des Sciences et Technologies, \\ Département de Biologie Végétale, Sénégal. \\ *Auteur correspondant ; E-mail : goudiabyarfang@gmail.com, Tél: + 221772090641
}

\section{REMERCIEMENTS}

Les auteurs remercient le Projet Bois Energie du Sénégal (BES) pour avoir financé les travaux. Nous remercions également l'Institut Sénégalais de Recherches Agricoles (ISRA) pour nous avoir donné une bourse de recherche.

\section{RESUME}

L'objectif de ce travail était d'évaluer l'effet du couvert et de la litière de Eucalyptus camaldulensis sur la diversité de la strate herbeuse. Pour atteindre cet objectif, un inventaire des herbacées sous et hors couvert a été réalisé. La litière sous Eucalyptus était quantifiée. Le pH du sol sous et couvert a été mesuré. Les résultats de l'inventaire floristique ont permis de dénombrer 46 espèces réparties dans 33 genres et 17 familles en zone sous couvert contre 39 espèces réparties dans 29 genres et 16 familles en zone hors couvert. Aucune différence significative n'est notée de l'indice de Shannon-Weaver trouvé entre les deux zones $(\mathrm{P}=0,07)$. Par contre, l'indice de régularité est plus élevé en zone sous couvert qu'en zone hors couvert $(\mathrm{P}=0,01)$. La quantité de feuilles collectées est plus importante en novembre $\left(55,22 \mathrm{~g} \mathrm{MS} / \mathrm{m}^{2}\right)$, janvier $\left(23,74 \mathrm{~g} \mathrm{MS} / \mathrm{m}^{2}\right)$ et mars $\left(30,36 \mathrm{~g} \mathrm{MS} / \mathrm{m}^{2}\right)$ qu'en juillet $\left(2,17 \mathrm{gMS} / \mathrm{m}^{2}\right)$ et septembre $\left(4,33 \mathrm{gMS} / \mathrm{m}^{2}\right)(\mathrm{P}=0,0001)$. L'analyse (Régression) a montré une corrélation très faibles entre la diversité et le $\mathrm{pH}$ sous et hors couvert. Cette étude a permis de mettre en évidence la biodiversité entre la zone sous et hors couvert de E. camaldulensis.

(C) 2017 International Formulae Group. All rights reserved.

Mots clés : Diversité spécifique, Herbacées, Diembéring, Sénégal. 


\title{
Influence of canopy and litter of Eucalyptus camaldulensis (dehn) on the specific diversity of herbaceous plants in the Diembéring area (Basse Casamance, Senegal)
}

\begin{abstract}
The objective of this study was to evaluate the effect of canopy and litter of Eucalyptus camaldulensis on the diversity of the grass stratum. To archeive this objectif, an inventory of herbaceous plants under and out of cover was carried out. The litter under Eucalyptus was quantified. The soil pH under and out the litter was measured. The floristic inventory results revealed 46 species, distributed in 33 genera and 17 families, in the zone under Eucalyptus cover, against 39 species spread in 29 genera and 16 families in zone not covered. There was no significant difference in the Shannon-Weaver index, between the two areas $(P=0.07)$. However, the regularity index is higher in the undercover area than in the non-covered area $(\mathrm{P}=0.01)$. The mass of collected leaves was greatest in November (55.22 $\mathrm{g}$ MS / $\left.\mathrm{m}^{2}\right)$, January $\left(23.74 \mathrm{~g} \mathrm{MS} / \mathrm{m}^{2}\right)$ and March $\left(30.36 \mathrm{~g} \mathrm{MS} / \mathrm{m}^{2}\right)$ than in July $\left(2.17 \mathrm{gMS} / \mathrm{M}^{2}\right)$ and September $\left(4.33 \mathrm{gms} / \mathrm{m}^{2}\right)(\mathrm{P}=0.0001)$. The analysis showed very weak correlations between diversity and $\mathrm{pH}$ under and out cover. This study allowed highlighting the biodiversity between the areas under and beyond cover.
\end{abstract}

(C) 2017 International Formulae Group. All rights reserved.

Keywords: Senegal, Diembering, Specific Diversity, herbaceous.

\section{INTRODUCTION}

Au cours de ces dernières décennies, on a assisté à un processus de dégradation généralisée des écosystèmes naturels. Cette dégradation aggravée par le contexte socioéconomique et pédoclimatique défavorable, s'est caractérisée par une réduction importante des formations végétales (Remigi et al., 2008). La forte pression anthropique exercée sur le milieu a eu comme corollaire la déforestation, la réduction de la durée des jachères, le surpâturage et la dégradation des ressources naturelles en général (Remigi et al., 2008).

Pour freiner cette déforestation et faire face aux besoins en bois, les politiques nationales de gestion des ressources naturelles sont de plus en plus orientées vers les plantations d'essences exotiques mais également locales à croissance rapide et à rendement élevé en produits ligneux. Ces plantations d'essences exotiques occupent de nos jours de vastes espaces au détriment des forêts naturelles qui sont en train de disparaitre à un rythme sans précédent (Ferreira et al., 2006). Cette conversion de forêts naturelles en plantations forestières exotiques impliquerait le remplacement d'un milieu à biodiversité stable et riche par une forêt artificielle presque mono-spécifique (Djégo et Sinsin, 2006). D'ailleurs, de nombreux chercheurs s'accordent à affirmer que le maintien d'une biodiversité élevée est plus favorisé par les essences indigènes que par des espèces introduites. L'introduction de nouvelles espèces aurait une incidence directe sur la conservation et la valorisation de la biodiversité floristique (Remigi et al., 2008). Ces dernières sont implantées loin de leurs aires de répartitions naturelles et, de ce fait, loin du cortège floristique qui a évolué avec elles (Sykes, 2001).

Le genre Eucalyptus est l'une des principaux genres forestiers plantés dans le monde (Nguyen et al., 2006). Sa croissance rapide, la diversité d'espèces disponibles et sa rusticité permettent d'établir rapidement une ressource ligneuse et de répondre à des besoins divers.

Cependant, l'intérêt de ces plantations est vivement remis en cause par certains chercheurs du fait qu'elles ont des effets supposés néfastes sur le sol et sur la diversité du couvert végétal (Pamela et al., 2003). 
L'absence d'études comparant la biodiversité des plantations d'eucalyptus à celle de plantations monospécifiques d'essences locales est à ce titre regrettable (Tassin et al., 2011). Les espèces de Eucalyptus introduites modifient les caractères physico-chimiques du sol et la luminosité arrivant au sous-bois (Peterken 2001). Eucalyptus camaldulensis produit en permanence de la litière qui se décompose lentement en nutriments et entraine des changements biochimiques du sol (Grierson et al., 2000). Ces impacts supposés négatifs sur la diversité végétale et sur le sol suscitent un intérêt grandissant pour les chercheurs. Au Sénégal, particulièrement en Casamance, aucune étude de l'impact de Eucalyptus camaldulensis sur la diversité de la strate herbeuse n'a été effectuée depuis son introduction comme essence de reboisement.

L'objectif de ce travail consiste à évaluer l'effet du couvert et de la litière de Eucalyptus camaldulensis sur la diversité de la strate herbeuse. Il est sous tendu par l'hypothèse scientifique suivante : «l'espèce Eucalyptus camaldulensis a des effets négatifs sur la diversité des herbacées».

\section{MATERIEL ET METHODES}

\section{Zone de l'étude}

L'étude a été conduite dans le département de Oussouye au Sud du pays, plus précisément dans la commune de Diembéring (Figure 1). Le relief est généralement plat avec de bas plateaux dominant à peine des dépressions servant à la riziculture. Le climat est de type Sudsoudanien côtier (Sagna, 2005). La moyenne pluviométrique annuelle est d'environ 1200 $\mathrm{mm}$ et la température moyenne est de $27^{\circ} \mathrm{C}$. Le climat est dominé par deux saisons : la saison sèche qui s'étale d'octobre à mai et la saison des pluies allant de juin à mi-octobre (Sagna, 2005). La végétation est arborée et arbustive de type savane boisée. Les espèces caractéristiques sont : Ceiba pentandra (L) Gaertn, Parinari exelsa Sabine, Adansonia digitata L., Parkia biglobosa (Jacq.) R.Br. ex G.Don, Khaya senegalensis (Desr.) A. Juss,
Faidherbia albida (Delile) A. Chev., et les palétuviers (Badji, 2016).

\section{Relevés de la végétation herbacée}

L'inventaire floristique a été conduit dans une plantation de Eucalyptus camaldulensis de 12 ha âgée de 8 ans. Il a été fait dans la zone sous couvert et hors couvert de la plantation de Eucalyptus camaldulensis. La hauteur moyenne des arbres composant la plantation est de $11 \mathrm{~m}$. Dans cette plantation, E. camaldulensis a été plantée en lignes parallèles avec un écart de $2 \mathrm{~m}$ entre les plantes sur une même ligne et de $10 \mathrm{~m}$ entre deux lignes soit une densité de 2400 arbres. Les relevés de végétation sous couvert ont été réalisés en suivant les lignes de la plantation. Les effets bordures ont été pris en compte lors de l'échantillonnage. Pour ce faire une ligne sur cinq (5) a été choisie et sur la ligne, un arbre sur deux a été également choisi. Au total, les relevés ont été effectués sur vingtcinq (25) arbres, soit cinq (5) arbres par ligne.

L'aire considérée pour l'inventaire sous couvert correspond à la projection verticale du houppier au sol. Ainsi, des carrés de $1 \mathrm{mx} 1 \mathrm{~m}$ ont été placés à $0 \mathrm{~cm}$ du tronc de l'arbre c'est-à-dire à $\mathrm{R}_{0}$ et à la limite de la zone de projection du houppier c'est-à-dire à $\mathrm{R}$ (rayon). Ces carrés ont été placés suivant l'orientation Est-Ouest et Nord-Sud soit 8 relevés par arbre.

Les relevés hors couvert ont été réalisés suivant la méthode des transects. Cinq transects orientés Est-ouest et Nord/Sud, donc transversaux, distants d'environ $20 \mathrm{~m}$ l'un de l'autre, ont été installés. Le long de chaque transect des relevés distants d'environ $5 \mathrm{~m}$ ont été effectués dans des placettes de $1 \mathrm{~m}^{2}$. Les coordonnées géographiques de chaque relevé ont été déterminées à l'aide du GPS afin de localiser les relevés. Au total, deux cent cinquante (250) relevés floristiques ont été réalisés dont cent cinquante (150) dans la zone sous couvert et cent (100) dans la zone hors couvert. Toutes les herbacées sous et hors couvert ont été inventoriées et identifiées sur le terrain et/ou au laboratoire, à l'aide de la 
flore du Sénégal (Berhaut, 1967) et des adventices tropicales (Merlier et Montegut, 1982). Les paramètres évalués sont la composition floristique et la diversité spécifique.

\section{Production de la litière sous Eucalyptus camaldulensis}

La litière produite au sein des plantations de Eucalyptus camaldulensis a été estimée à l'aide de la méthode de pesée de la matière sèche. Vingt (20) trappes ont été conçues en s'inspirant du modèle de Brown (1984) et placées à l'intérieur de la plantation. C'est un outil en forme de panier de $50 \mathrm{~cm}$ x $50 \mathrm{~cm}$ d'ouverture $\times 25 \mathrm{~cm}$ de fond. Ses parois (fond et flancs) ont été recouvertes d'une moustiquaire en plastique à maille de 1 $\mathrm{mm} \times 1 \mathrm{~mm}$. La collecte a été réalisée tous les deux mois durant une année entière. La litière collectée a été séchée à l'étuve à $70{ }^{\circ} \mathrm{C}$ pendant 72 heures. Elle a ensuite été triée pour séparer les différents éléments végétaux (feuilles, inflorescences, brindilles, écorces...) des autres éléments tels que les restes d'insectes, les plumes d'oiseaux.

\section{Mesures de pH}

Des prélèvements de sols ont été effectués sous et hors du couvert de $E$. camaldulensis. Sept (7) échantillons ont été prélevés dans la zone sous et hors couvert. Pour chaque échantillon effectué, cinq points (5) de prélèvements ont constitué un échantillon composite. A chaque échantillon sous couvert correspond un échantillon témoin (HC), supposé non influencer par $E$. camaldulensis. Les sols ont été prélevés à l'horizon 0-10 cm. Les solutions de sols ont été préparées au laboratoire d'Agroforesterie et les mesures ont été effectuées avec un $\mathrm{pH}$ mètre.

\section{Analyse des données}

Les données de l'inventaire et de la litière colletée ont été traitées avec le tableur Excel. Les analyses de variance (ANOVA) et la régression linéaire ont été faites avec le logiciel XLSTAT 2014 Version 16.5.03. Le niveau d'organisation du peuplement herbacé a été évalué à l'aide de l'indice de diversité de Shannon-Weaver (H'), de l'indice de régularité $(\mathbf{R})$ et de la diversité maximale (Hmax).

\section{Indice de Shannon-Weaver ( $H^{\prime}$ )}

L'indice de Shannon est exprimé en bit et varie généralement de 0 à 5 . Elle varie en fonction du nombre d'espèces recensées et des effectifs de chacune de ces espèces; ce qui rend son utilisation moins pertinent pour la comparaison de deux milieux à richesse spécifique très dissemblable. De ce fait, il paraît plus indiqué d'utiliser l'équitabilité ou indice de régularité, qui est une portion de la valeur maximale que cet indice aurait si les individus étaient distribués de façon totalement égale parmi les espèces. Il apparaît comme un terme de comparaison plus rigoureux (Devineau et al., 1984). L'indice de Shannon-Weaver se calcule par la formule suivante :

$H^{\prime}=-\Sigma P^{*} \log 2 *{ }^{*} \mathbf{p i}$

$\mathrm{Pi}=\mathrm{ni} / \mathrm{N}$ représente l'abondance relative de l'espèce $\mathrm{i}$

ni est l'effectif de la population de l'espèce i

$\mathrm{N}$ est la somme des effectifs des $\mathrm{S}$ espèces constituant le peuplement considéré

\section{Diversité maximale (H max):}

Hmax $=\log 2 * S, S=$ effectif total des espèces

Indice de régularité (Equitabilité) (R) :

L'indice de régularité varie entre 0 et 1. Il tend vers 0 lorsque la quasi-totalité des effectifs appartient à une seule espèce et tend vers 1 lorsque chacune des espèces est représentée par le même nombre d'individus (Ramade et al., 1990). L'indice de régularité exprime donc la répartition des individus entre les différentes espèces du peuplement. Il a été calculé à l'aide de la formule suivante:

$\mathbf{R}=\mathbf{H}^{\prime} / \mathbf{H m a x}$, avec, Hmax $=\log 2 \mathrm{~S}$ la diversité spécifique maximale de Shannon. 


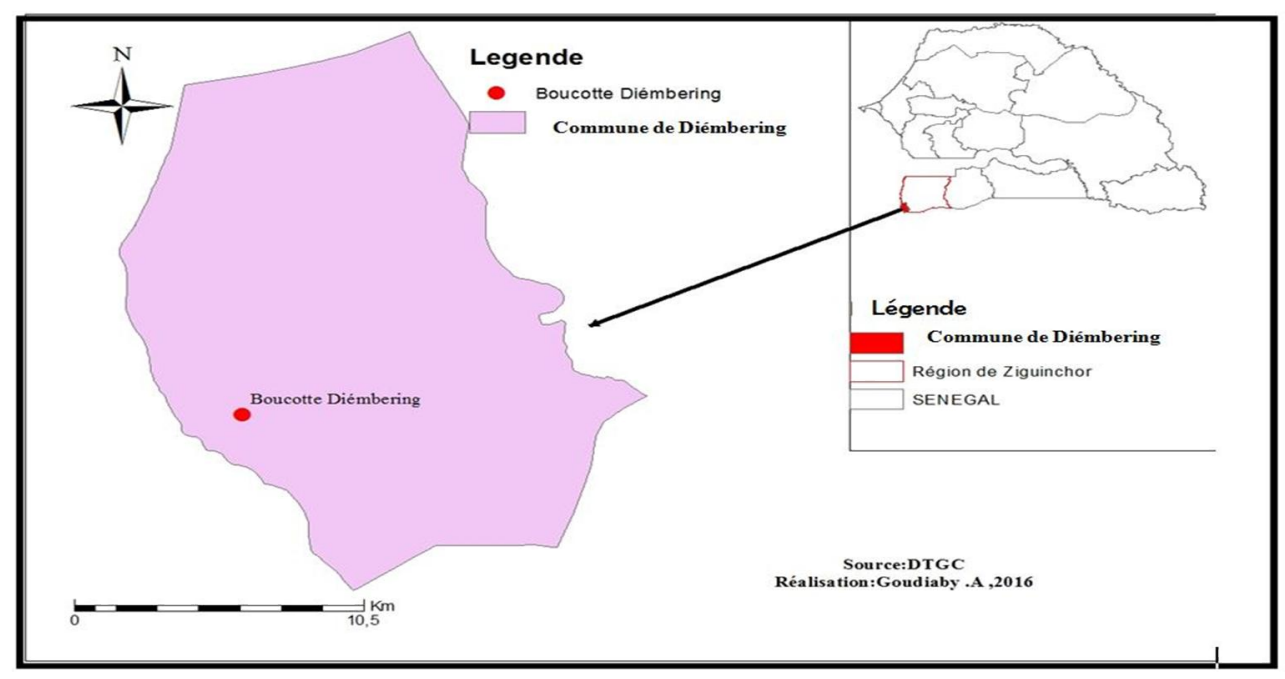

Figure 1: Carte de localisation de la zone d'étude.

\section{RESULTATS \\ Composition floristique}

L'inventaire floristique des herbacées sous et hors couvert a permis de recenser au total 49 espèces réparties dans 35 genres et 19 familles (Tableau 1). En effet, 46 espèces réparties dans 33 genres et 17 familles ont été dénombrées dans la zone sous couvert contre 39 espèces réparties dans 29 genres et 16 familles dans la zone hors couvert. Les espèces les plus importantes dans la zone sous couvert appartiennent à la famille des Poaceae (19,56\%), les Fabaceae (15,21\%), les Convolvulaceae (10,86\%), Malvaceae $(8,69 \%)$, les Rubiaceae $(6,52 \%)$ et les Tiliaceae (6,52\%). Elles représentent 60,84\% des espèces inventoriées sous couvert. Celles qui sont les plus importantes dans la zone hors couvert sont les Poaceae $(17,94 \%)$, les Fabaceae (12,52\%), les Convolvulaceae (12,52\%), les Rubiaceae (7,69\%), les Tiliaceae (7,69\%) et les Malvaceae (7,69\%). Celles qui sont les plus importantes dans la zone hors couverte sont les Poaceae $(17,94 \%$ des espèces), les Fabaceae $(12,52 \%$ des espèces), les Convolvulaceae $(12,52 \%$ des espèces), les Rubiaceae, les Tiliaceae et les Malvaceae (7,69\% des espèces).

\section{Espèces caractéristiques et espèces indifférentes}

Les espèces recensées peuvent être distinguées en deux catégories: celles présentes exclusivement, soit dans la zone sous couvert soit dans celle hors couvert dites espèces caractéristiques ou exclusives et celles présentes dans les deux zones dites espèces indifférentes ou communes. Le groupe des espèces indifférentes renferme 36 espèces tandis celui des espèces caractéristiques comporte 10 espèces pour la zone sous couvert et 3 espèces pour la zone hors couvert (Tableau 1). Les espèces caractéristiques sont nettement plus nombreuses sous le couvert de l'arbre que hors couvert. Sous le couvert, les espèces sont, dans leur grande majorité, des dicotylédones (Acanthaceae, Aizoaceae, Capparideae, Convolvulaceae, Sterculiaceae Fabaceae, Malvaceae). Dans les milieux découverts, les espèces sont représentées notamment par des Poaceae à feuilles étroites, enroulées. Les espèces indifférentes sont nettement plus nombreuses que celles dites exclusives ou caractéristiques. Dans notre étude, nous avons répertorié 15 familles (Tableau 1). 


\section{Diversité spécifique}

Les analyses de variance (ANOVA) sur l'indice de diversité de Shannon-Weaver $\left(\mathrm{H}^{\prime}\right)$ et sur la diversité maximale (Hmax) indiquent qu'il n'y a aucune différence significative entre les valeurs obtenues dans la zone sous couvert et celle obtenue dans la zone hors couvert $(P=0,07$, Fisher LSD au seuil de $5 \%$ pour H' et $\mathrm{P}=0,33$, Fisher LSD au seuil de 5\% pour Hmax). Cependant, une différence hautement significative est notée entre l'indice de régularité obtenue dans la zone sous couvert $(0,8)$ et celle obtenue dans la zone hors couvert $(0,7)(\mathrm{P}=0,001$,$) . Le$ peuplement herbacé sous Eucalyptys camaldulensis est plus diversifié et donc plus stable que celui hors couvert (Tableau 2).

\section{Production de la litière sous Eucalyptus camaldulensis}

La quantité totale de la litière produite dans les 20 trappes placées dans la plantation de Eucalyptus camaldulensis est présentée au niveau du Tableau 3.

Les analyses de variances (ANOVA) des données relatives à la masse de feuilles tombée pendant la période de l'étude montrent qu'il y a une différence très hautement significative entre la masse de feuilles collectée au mois de novembre $(55,22$ $\left.\mathrm{gMS} / \mathrm{m}^{2}\right)$ et celle collectée pendant les mois de janvier $\left(23,74 \mathrm{gMs} / \mathrm{m}^{2}\right)$, mars $(30,36$ $\left.\mathrm{gMS} / \mathrm{m}^{2}\right)$, juillet $\left(2,17 \mathrm{gMS} / \mathrm{m}^{2}\right)$ et de septembre $\left(4,33 \mathrm{gMS} / \mathrm{m}^{2}\right)(\mathrm{P}=0,0001$, Ficher LSD au seuil de 5\%).

Quant aux brindilles, l'analyse montre également une différence très hautement significative $(P=0,0001$, Fisher LSD au seuil de 5\%) entre la masse de brindilles collectée au mois de janvier par rapport au mois de mars $\left(5,13 \mathrm{gMS} / \mathrm{m}^{2}\right)$, juillet $\left(0,80 \mathrm{gMSm}^{2}\right)$ et novembre $(2,08$ $\left.\mathrm{gMS} / \mathrm{m}^{2}\right)$. Cette différence n'est par contre pas significative pour les masses des inflorescences et des écorces récoltés pendant toute cette période (Tableau 3 ).

\section{Déterminisme de diversité de la végétation herbacée sous et hors Eucalyptus camaldulensis \\ pH du sol de la zone sous et hors couvert}

L'analyse de variance ( ANOVA) sur le $\mathrm{pH}$ montre une différence très hautement significative entre la zone sous et couvert $(\mathrm{P}=0,0001$, Fisher LSD au seuil de $5 \%)$ (Tableau 4).

\section{Influence du pH sur la diversité spécifique des herbacées sur la zone sous couvert}

La Figure 2 montrant l'influence du $\mathrm{pH}$ sur la diversité révèle un coefficient de détermination $\left(\mathrm{R}^{2}\right)$ de 0,0066 et un coefficient de corrélation $(\mathrm{R})$ de 0,08 . Ce résultat indique une très faible corrélation entre le $\mathrm{pH}$ et la diversité. Le $\mathrm{pH}$ du sol n'a donc pas d'effets significatifs sur la diversité spécifique et la stabilité du peuplement herbacé dans la zone d'étude.

\section{Influence du pH sur la diversité spécifique des herbacées en zone hors couvert \\ La Figure 3 montre un coefficient de détermination $\left(\mathrm{R}^{2}\right)$ de 0,1106 et un coefficient de corrélation $(\mathrm{R})$ de 0,341 . Ce résultat indique que la corrélation entre le $\mathrm{pH}$ et la diversité spécifique des herbacées hors couvert de E. camadulensis est faible. Elle est toutefois plus importante qu'en zone couverte.}

Tableau 1 : Composition des espèces en fonction des zones sous couvert et hors couvert.

\begin{tabular}{lcccc}
\hline Familles & Genres & Espèces & SC & HC \\
\hline Acanthaceae & Peristrophe & Peristrophe bicalyculata (Retz) Nees. & + & - \\
\hline Aizoaceae & Mollugo & Mollugo nudicaulis Lam., & + & - \\
\hline $\begin{array}{l}\text { Apocynaceae- } \\
\text { Asclepiadoideae }\end{array}$ & Leptadenia & Leptadenia hastata (Pers.) Decne. & - & + \\
\hline
\end{tabular}


A.O.K. GOUDIABY et al. / Int. J. Biol. Chem. Sci. 11(4): 1471-1483, 2017

\begin{tabular}{|c|c|c|c|c|}
\hline Asclepiadaceae & Pergularia & Pergularia daemia (Forssk.) Chiov & + & + \\
\hline \multirow{2}{*}{ Caesalpiniaceae } & \multirow{2}{*}{ Cassia } & Cassia obtusifolia L. & + & + \\
\hline & & Cassia mimosoides L. & + & + \\
\hline Capparidaceae. & Cleome & Cleome coeruleo-rosea Gilg et Benedict & + & - \\
\hline \multirow{2}{*}{ Commelinaceae } & \multirow{2}{*}{ Commelina } & Commelina benghalensis L., & + & - \\
\hline & & Commelina forskalaei Vahl. & + & + \\
\hline Compositae & Acanthospermum & Acanthospermum hispidum DC., & + & + \\
\hline \multirow{5}{*}{ Convolvulaceae } & \multirow{3}{*}{ Ipomoea } & Ipomoea eriocarpa R.BR. & + & + \\
\hline & & Ipomoea coptica (L.) Roth & + & + \\
\hline & & Ipomoea sp & + & + \\
\hline & \multirow[b]{2}{*}{ Merremia } & Merremia aegyptiaca (L.) Urban & + & + \\
\hline & & Merremia tridentata (L.) Hall. & + & - \\
\hline \multirow{2}{*}{ Cyperaceae } & \multirow{2}{*}{ Cyperus } & Cyperus amabilis Vahl. & + & + \\
\hline & & Cyperus rotundus L., & + & + \\
\hline Euphorbiaceae & Euphorbia & Euphorbia hirta L. & - & + \\
\hline \multirow{7}{*}{ Fabaceae } & \multirow{2}{*}{ Tephrosia } & Tephrosia linearis (Willd) Pers & + & + \\
\hline & & Tephrosia purpurea Pers. & + & - \\
\hline & \multirow[b]{2}{*}{ Indigofera } & Indigofera hirsuta $L$. & + & + \\
\hline & & Indigofera pilosa Poir. & + & + \\
\hline & Sesbania & Sesbania pachycarpa DC. & + & + \\
\hline & \multirow{2}{*}{ Crotalaria } & Crotalaria goreensis Guill.et Perr & + & - \\
\hline & & Crotalaria retusa $L$. & + & + \\
\hline \multirow{9}{*}{ Poaceae } & Dactyloctenium & Dactyloctenium aegyptium Beauv & + & + \\
\hline & Digitaria & Digitaria horizontalis Willd. & + & + \\
\hline & \multirow[b]{2}{*}{ Eragrostis } & Eragrostis ciliaris var.ciliaris (L.) R. Br. & + & + \\
\hline & & $\begin{array}{c}\text { Eragrostis tremula (Lamarck) Hochstetter ex } \\
\text { Steudel }\end{array}$ & - & + \\
\hline & Brachiaria & Brachiaria villosa (Lam.) A. Camus & + & + \\
\hline & Setaria & Setaria pumila (Poir.) Roem. \& Schult & + & - \\
\hline & \multirow{2}{*}{ Andropogon } & Andropogon gayanus Kunth & + & + \\
\hline & & Andropogon pseudapricus Stapf. & + & + \\
\hline & Ischaemum & $\begin{array}{l}\text { Ischaemum afrum (J.F. Gmelin) Dandy (J.F. } \\
\text { Gmelin) Dandy }\end{array}$ & + & + \\
\hline \multirow{2}{*}{ Lamiaceae } & \multirow{2}{*}{ Hyptis } & Hyptis spicigera Lam. & + & + \\
\hline & & Hyptis suaveonlens (L.) Poit. & + & + \\
\hline
\end{tabular}


A.O.K. GOUDIABY et al. / Int. J. Biol. Chem. Sci. 11(4): 1471-1483, 2017

\begin{tabular}{|c|c|c|c|c|}
\hline \multirow{4}{*}{ Malvaceae } & \multirow{2}{*}{ Hibiscus } & Hibiscus asper Hook. $f$. & + & + \\
\hline & & Hibiscus hasirikus Berhaut & + & + \\
\hline & Sida & Sida alba $L$ & + & + \\
\hline & Urena & Urena lobata L. & + & - \\
\hline \multirow[t]{2}{*}{ Mimosaceae } & Mimosa & Mimosa pudica $L$. & + & + \\
\hline & Oldenlandia & Oldenlandia corymbosa L., & + & + \\
\hline \multirow[t]{2}{*}{ Rubiaceae } & Mitracarpus & Mitracarpus villosus (Sw.) DC. & + & + \\
\hline & Spermacoce & Spermacoce stachydea (DC.) Hutch. Et Dalz. & + & + \\
\hline \multirow{2}{*}{ Sterculiaceae } & Walteria & Walteria indica $L$ & + & - \\
\hline & Melhania & Melhania ovata (cav) Spreng & + & + \\
\hline \multirow{3}{*}{ Tiliaceae } & \multirow{2}{*}{ Corchorus } & Corchorus olitorius L. & + & + \\
\hline & & Corchorus tridens $L$. & + & + \\
\hline & Triumpheta & Triumpheta pentandra A. Rich., & + & + \\
\hline
\end{tabular}

Tableau 2: Indices de diversité dans les zones sous et hors couvert.

\begin{tabular}{lccc}
\hline Zone & $\begin{array}{c}\text { Indice de Shannon- } \\
\text { Weaver }\left(\mathbf{H}^{\prime}\right)\end{array}$ & $\begin{array}{c}\text { Diversité maximale } \\
(\mathbf{H m a x})\end{array}$ & $\begin{array}{c}\text { Indice de } \\
\text { régularité }(\mathbf{R})\end{array}$ \\
\hline Sous couvert & 2,3 & $3 \mathrm{a}$ & $0,8 \mathrm{~b}$ \\
Hors couvert & 2,3 & $3 \mathrm{a}$ & $0,7 \mathrm{a}$ \\
Probabilité et & $0,07^{\mathrm{ns}}$ & $0,33^{\mathrm{ns}}$ & $0,001^{\mathrm{n}}$ \\
significativité & & & \\
\hline Sur une même colonne, les valeurs avec des lettres différentes sont significativement différentes à P $<0,05$ selon le test de \\
Fisher (LSD). NS = Non Significative $; \mathrm{N}=$ Significative.
\end{tabular}

Tableau 3 : Evolution de la masse de Matière sèche $\left(\mathrm{g} \mathrm{MS} / \mathrm{m}^{2}\right)$ des différentes composantes de la litière en fonction du temps.

\begin{tabular}{lcccc}
\hline Mois & Feuilles & Brindilles & Inflorescence & Ecorce \\
\hline juillet, 2015 & $2,17 \mathrm{c}$ & $0,80 \mathrm{c}$ & $0,028 \mathrm{a}$ & $0,56 \mathrm{a}$ \\
septembre, 2015 & $4,33 \mathrm{c}$ & $0,55 \mathrm{c}$ & $0.07 \mathrm{a}$ & $0,06 \mathrm{a}$ \\
novembre, 2015 & $55,22 \mathrm{a}$ & $2,08 \mathrm{c}$ & $0,8 \mathrm{a}$ & $0,48 \mathrm{a}$ \\
janvier, 2016 & $23,74 \mathrm{bc}$ & $10,56 \mathrm{a}$ & $0,0005 \mathrm{a}$ & $0,06 \mathrm{a}$ \\
mars, 2016 & $30,36 \mathrm{~b}$ & $5,13 \mathrm{~b}$ & $0,07 \mathrm{a}$ & $0,12 \mathrm{a}$ \\
mai, 2016 & $4,14 \mathrm{~b}$ & $0,67 \mathrm{c}$ & $0,06 \mathrm{a}$ & $0,44 \mathrm{a}$ \\
\hline Probabilité et significativité & $0,0001^{\mathrm{n}}$ & $0,0001^{\mathrm{n}}$ & $0,37^{\mathrm{ns}}$ & $0,64{ }^{\mathrm{ns}}$ \\
\hline $\begin{array}{l}\text { Sur une même ligne, les valeurs avec des lettres différentes sont significativement différentes à P }<0,05 \text { selon le test de Fisher } \\
\text { (LSD). NS = Non Significative ; } \mathrm{N} \text { Significative }\end{array}$ & &
\end{tabular}


Tableau 4 : variation entre zone sous et hors couvert.

\begin{tabular}{ccc}
\hline \multirow{2}{*}{$\mathbf{p H}$} & \multicolumn{3}{c}{$\mathbf{\text { SC }}$} & HC \\
\cline { 2 - 3 } & \multicolumn{5}{c}{$\mathbf{5 , 5} \mathbf{a}$} & $\mathbf{6 , 0 2} \mathbf{b}$ \\
\hline Probabilité et significativité & \multicolumn{2}{c}{$0,0001^{\mathrm{n}}$} \\
\hline
\end{tabular}

Sur une même ligne, les valeurs avec des lettres différentes sont significativement différentes à $\mathrm{P}<0,05$ selon le test de Fisher (LSD). NS = Non Significative ;

$\mathrm{N}=$ Significative.

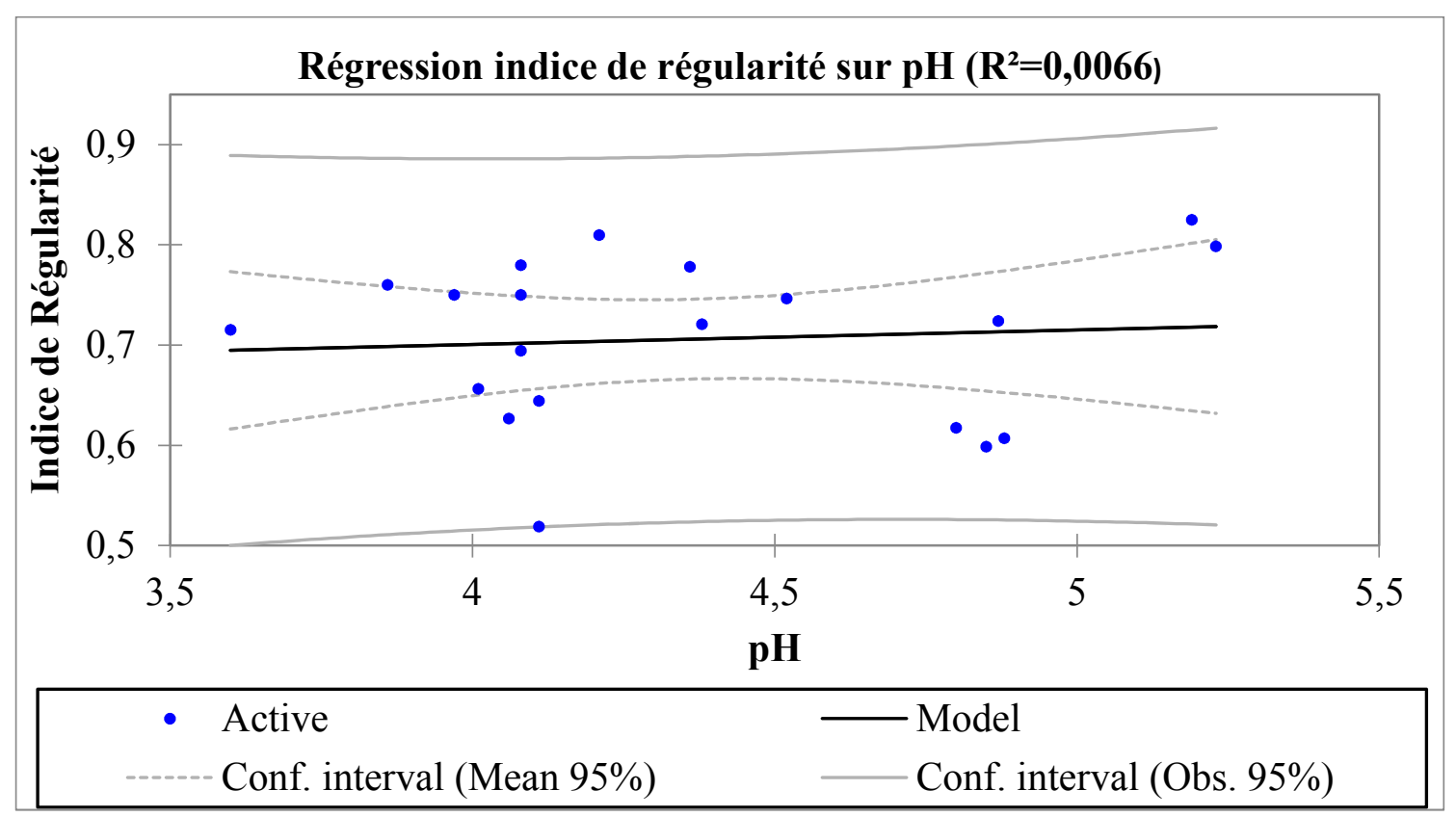

Figure 2: Relation entre la diversité (indice de régularité) et le $\mathrm{pH}$ dans la zone sous couvert. 


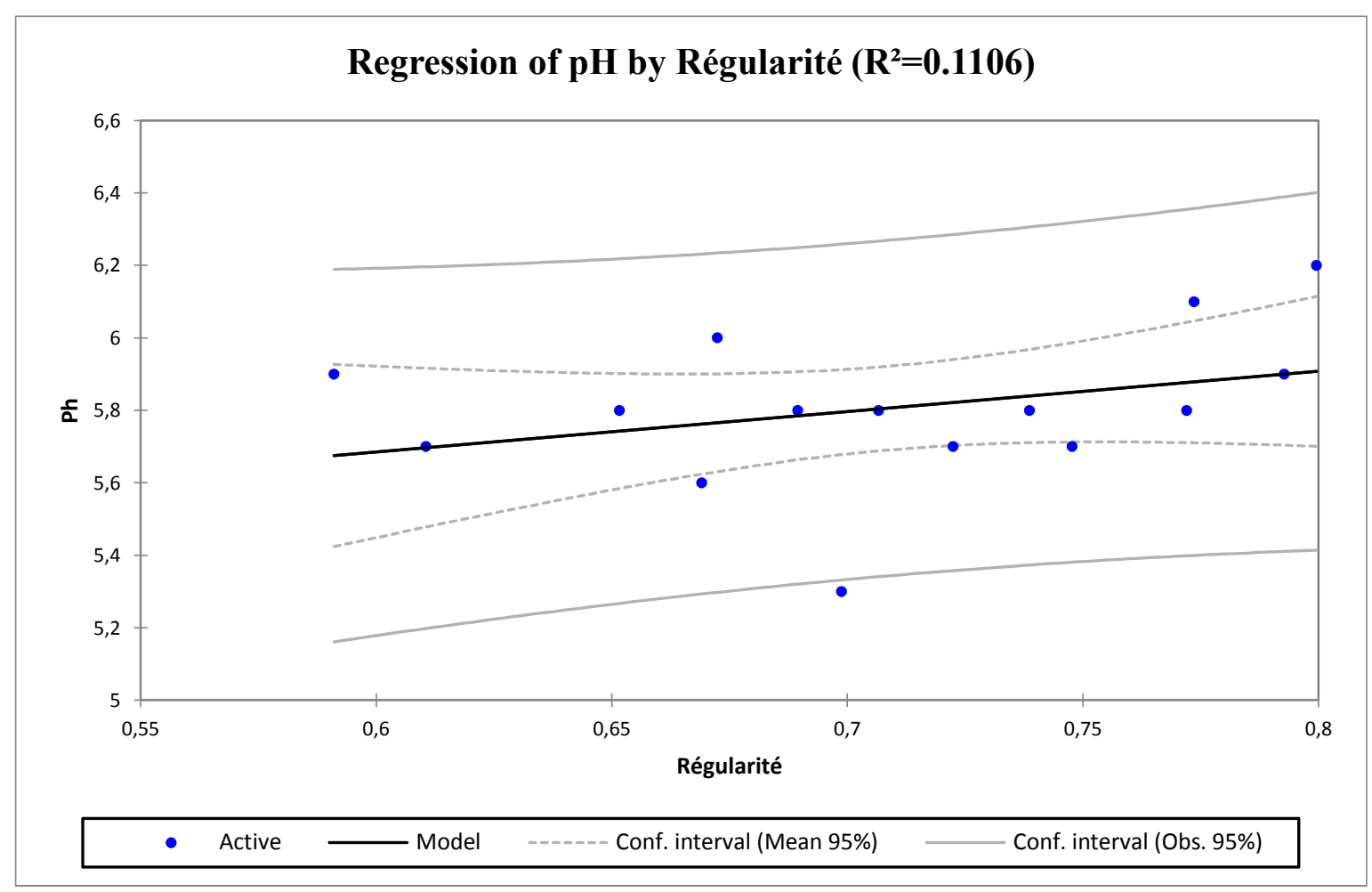

Figure 3 : Relation entre la diversité (indice de régularité) et le pH dans la zone hors couvert.

\section{DISCUSSION}

\section{Composition de la flore des herbacées}

Il ressort de l'analyse des résultats de l'inventaire floristique des herbacées dans les zones sous et hors couvert que ce sont les Poaceae (07 genres, 09 espèces), les Fabaceae (04 genres, 07 espèces), les Malvaceae (03 genres, 04 espèces), les Rubiaceae (03 genres, 03 espèces), les Convolvulaceae (02 genres, 05 espèces) qui sont les plus représentées. Cette bonne représentativité de ces familles pourrait s'expliquer par leur adaptation aux conditions du milieu. Ces résultats corroborent ceux de Diallo et al. (2015) qui, dans leur étude, ont montré la prédominance des espèces appartenant à ces familles sous le couvert des arbres.

L'analyse des résultats sur la richesse et la diversité spécifique a montré que la zone sous couvert végétal était riche de 46 espèces alors que celle de la zone hors couvert était de 39 espèces. Cette richesse de la flore herbacée sous couvert végétal serait due à la sylviculture c'est-à-dire à la façon dont les arbres sont plantées, et à l'âge des individus de Eucalyptus camaldulensis. Ce résultat confirme celui de Rajvanshi (1983) qui indique un ordre de grandeur de répartition semblable des espèces sous couvert de Eucalyptus camaldulensis (65 espèces) et hors couvert végétal (37 espèces) en Inde. De même, certains auteurs ont montré qu'en zones semi-arides, le nombre d'espèces liées au couvert ligneux est nettement plus élevé que le nombre d'espèces inféodées aux milieux découverts (Akpo et al., 2004 ; Diallo et al., 2015).

Ces résultats infirment ceux de Djègo et Sinsin (2006) qui ont montré que dans le 
sous-bois des plantations monospécifiques de E. camaldulensis ou en mélange avec Acacia auriculiformis, la richesse spécifique est de 34 espèces pour E. camaldulensis et 99 espèces pour les peuplements de Casuarina equisetifolia en culture pure ou mélangé à Acacia auriculiformis. Tassin et al. (2011) pensent qu'au au-delà des effets allélopathiques, l'abondance du sous-bois dépend de l'état des ressources hydriques et de leur accès par les racines. Le $\mathrm{pH}$ acide de la litière de E. camaldulensis et probablement la présence de substances toxiques phénoliques ou volatiles contenues dans les feuilles seraient préjudiciables à la richesse spécifique de leur sous-bois. Par ailleurs, la concurrence et le développement important de racines traçantes par les pieds de E. camaldulensis sont des facteurs limitant pour la végétation de sous-bois et les cultures avoisinantes dans le cas où l'eau serait peu abondante.

\section{Diversité spécifique des herbacées}

En référence à la sylviculture, Bassou, (2003) note que quand les Eucalyptus sont plantés de façon ouverte, elles donnent un écosystème plus riche. Cette tendance de la répartition de la flore herbacée sous et hors couvert végétal est confirmée par l'indice de régularité significativement plus élevé sous les pieds de Eucalyptus camaldulensis $(0,8)$ qu'en dehors de leur couvert $(0,7)$. Toutefois, ce résultat est différent de celui de Djégo et Sinsin (2006) qui ont trouvé un indice de régularité de 0,47 sous les plantations de $E$. camaldulensis et 0,57 sous Casuarina equisetifolia au Bénin.

\section{Production de la litière sous Eucalyptus camaldulensis}

Les résultats des analyses statistiques des données sur la litière de E. camaldulensis montrent qu'elle est essentiellement constituée de feuilles (84,18\%). Cette production assez importante durant les mois de novembre, janvier et mars serait due à la période de sénescence de beaucoup d'arbres dont Eucalyptus camaldulensis. La quantité d'inflorescences et d'écorces est quasi-nulle dans cette litière. Ces résultats confirment ceux de Djègo et Sinsin (2006) qui ont montré que la litière de Eucalyptus camaldulensis est constituée essentiellement de feuilles (64 à $80 \%$ ).

\section{Déterminisme de diversité de la végétation herbacée sous Eucalyptus camaldulensis}

Les résultats des analyses sur les tests de corrélation entre la diversité (indice de régularité) et le $\mathrm{pH}$ et entre la diversité et la litière produite par les plantations de Eucalyptus camaldulensis montrent que les facteurs explicatifs supposés sont très faiblement corrélés à ceux expliqués. La diversité est donc très faiblement influencée par ces deux facteurs explicatifs

Cependant, Djégo et Sinsin (2006) ont montré également que le $\mathrm{pH}$, la masse de la litière et la luminosité présentent des effets significatifs sur la diversité spécifique du sous-bois.

\section{Conclusion}

Cette étude a permis de mettre en évidence la composition floristique des herbacées sous et hors couvert. Elle a montré que la flore sous Eucalyptus camaldulensis est plus riche que celle hors couvert végétal. L'inventaire floristique révèle que ce sont les Poaceae, les Fabaceae, les Convolvulaceae, les Malvaceae, les Rubiaceae et les Tiliaceae qui sont les plus représentatives dans la zone sous couvert. Ces familles représentent $60,84 \%$ des espèces inventoriées dans cette zone. Celles qui sont les plus importantes dans la zone hors couvert sont les Poaceae, les Fabaceae, les Convolvulaceae, les Rubiaceae, les Tiliaceae et les Malvaceae. Ces espèces appartiennent à 50,69\% des familles. Elle révèle que cette zone sous couvert était 
beaucoup plus stable que la zone hors couvert. Les données sur la litière ont indiqué que celle-ci était essentiellement constituée de feuilles $(84,14 \%)$. La régression entre la diversité spécifique et le $\mathrm{pH}$ en zone sous et hors couvert a permis de voir que leur corrélation est très faible.

\section{CONFLIT D'INTERETS}

Les auteurs déclarent, à l'unanimité, qu'il n'y a aucun conflit d'intérêt qui serait liée au présent article.

\section{CONTRIBUTIONS DES AUTEURS}

AOKG a contribué à la mise en place du protocole de recherche, l'inventaire de la végétation herbacée sous et hors couvert de Eucalyptus camaldulensis, l'analyse des données et la rédaction de l'article. SDS a coencadré les travaux de la thèse dans lequel ce papier est tiré, contribué à la rédaction de l'approche méthodologique de recherche, à la collecte, à l'analyse des données et à la rédaction de l'article. IC a contribué à la collecte aux traitements statistiques des données, collaborateur dans la rédaction de l'article. SD a collaboré dans la collecte, le traitement statistique des données et la rédaction de l'article. NN a contribué à la collecte aux traitements statistiques des données et à la rédaction de l'article. IN a dirigé les travaux, et a défini le sujet et a orienté les travaux de recherche.

\section{REFERENCES}

Akpo LE, Grouzis M. 2004. Interactions arbre/herbe en bioclimat semi-aride : influence de la pâture. Sécheresse, 15(3): 253-61.

Asseo M, Kawalec A, Lefebvre L. 1992. Bénin, revue du secteur agricole. La Banque Mondiale. Rap. $\mathrm{N}^{\circ}$ 10709-BEN. Ambassade Royale de Danemark Cotonou, Béninp, p. 58.
Badji M. 2016. Caractérisation et évaluation de la biomasse ligneuse et de la séquestration du carbone dans les plantations d'Eucalyptus camaldulensis Dehn., du projet CBED dans le département d'Oussouye. Mémoire de Master, Université Assane Seck de Ziguinchor, p. 59.

Bassou D. 2003. Impact des plantations d'eucalyptus sur la diversité floristique : Etude de cas dans le Lauragais, Rapport pour AFOCEL, p. 34.

Bara TS. 1986. Relacio Eucalipta-suelo de las zonas humedas de la peninsula iberica, $\mathrm{p}$. 17.

Berhaut J. 1967. Flore du Sénégal (deuxième édition plus complète avec les forêts humides de Casamance). Éditions Clairafrique.

Brown MS. 1984. Mangrove litter production and dynamics. In the Mangrove Ecosystem: Research Methods, Snedaker SC, Snedaker JG (eds). Unesco : Bungay, United Kingdom; 231-237.

Diallo MD, Ndiaye O, Diallo A, Saleh MM, Bassène C,Wood AS, Diop A, Guisse A. 2015. Influence de la litière foliaire de cinq espèces végétales tropicales sur la diversité floristique des herbacées dans la zone du Ferlo (Senegal). Int. J. Biol. Chem. Sci., 9(2): 803-814. DOI: 10.4314/ijbcs.v9i2.20

Devineau JL, Lecoudier C, Vatttoux R. 1984. Evolution de la diversité spécifique du peuplement ligneux dans une succession préforestière de colonisation d'une savane protégée des feux (Lamto, Côted'Ivoire). Conservatoire et Jardin Botanique de Genève, 39(1): 103-133.

Djègoa J, Sinsina B. 2006. Impact des espèces exotiques plantées sur la diversité spécifique des phytocénoses de leur sous-bois. Syst. Geogr. Pl., 76: 191-209. DOI : AR00036377 
Duponnois R, Founoune H, Masse D, Pontanier R. 2005. Inoculation of Acacia holosericea with ectomycorrhizal fungi in a semi-arid site in Senegal: growth response and influences on the mycorrhizal soil infectivity after 2 years plantation. Forest Ecology and Management, 207: 351-362.

Ferreira V, Elosegi A, Gulis V, Pozo J, Graça MAS. 2006. Eucalyptus plantations affect fungal communities associated with leaf-litter decomposition in Iberian streams. Arch. Hydrobiol., 166 (4) 467490. DOI: $\quad 10.1127 / 0003$ 9136/2006/0166-0467.

Grierson PF, Adams MA. 2000. Plant species affect acid phosphatase, ergosterol and microbial $P$ in a Jarrah (Eucalyptus marginata Donn ex Sm.) forest in southwest Australia. Soil Biol. Biochem., 32:1817-1827. DOI: 10. 1038/srep36672

Merlier H, Montegut J. 1982. Les Adventices Tropicales. ORSTOM-GERDAT-ENSH; p. 491.

Ndiaye O, Aly D, Stephen AW, Guissé A. 2014. Structural Diversity of Woody Species in the Senegalese Semi-Arid Zone - Ferlo. American Journal of Plant Sciences, 5: 416-426. DOI: http://dx.doi.org/10.4236/ajps.2014.5305 5

Nguyen N, Chaste B. 2006. Eucalyptus et environnement Rapport AFOCEL, p. 6.

Pamela J, Pender J. 2003. The role of trees for sustainable management of less favored lands: the case of Eucalyptus in Ethiopia. For. Policy Econ., 5: 83-95. DOI: 10.1016/S1389-9341(01)00078-8
Peterken GF. 2001. Ecological effects of induced tree species in Britain. Forest Ecology and Management, 141: 31-42. DOI: 10. 1111/j.1469-8137.2008.02579. $\mathrm{x}$

RAMADE F. MC GRAW H. 1990. Eléments d'écologie-Ecologie fondamentale, 185200.

Rajvanshi A, Soni S, Kukreti UD, Srivastara MM. 1983. A Comparaitive study of undergrowth of sal forest and Eucalyptus plantation of Golatappar-Dehra Dun during rainy season. Indian Journal of Forestory., $\quad$ 6(2): 117-119. DOI : 001152663

Remigi P, Faye A, Kane A, Deruaz M, Thioulouse J, Cissoko M, Prin Y, Galiana A, Dreyfus B. 2008.The Exotic Legume Tree Species Acacia holosericea Alters Microbial Soil Functionalities and the Structure of the Arbuscular Mycorrhizal Community. Appl. Environ. Microbiol., 74(5): 14851493. DOI: 10.1128/AEM.02427-07

Sagna P. 2005. Dynamique du climat et son evolution récente dans la partie ouest de l'Afrique occidentale. Thèse, Université Cheikh Anta DIOP de Dakar, p. 790.

Sykes MT. 2001. Modelling the potential distribution and community dynamics of lodgepole pine (Pinus contorta Dougl. Ex. Loud.) in Scandinavia. Forest Ecology and Management, 141: 69-84.

Tassin J, Missamba-Lola AP, Marien JN. 2011. Biodiversité des plantations d'eucalyptus. Bois et Forêts des Tropiques, 309(3): 27. 